\title{
Motivation Improves Working Memory by Two Processes: Prioritisation and Retrieval Thresholds
}

John P. Grogan ${ }^{1}$, Govind Randhawa ${ }^{1}$, Minho Kim² ${ }^{2}$ \& Sanjay G. Manohar ${ }^{1}, 2$.

1. Nuffield Department of Clinical Neurosciences, University of Oxford, UK

2. Department of Experimental Psychology, University of Oxford, UK

\section{Abstract}

Motivation can improve performance when the potential rewards outweigh the cost of effort expended. In working memory (WM), people can prioritise rewarded items at the expense of unrewarded items, suggesting a fixed memory capacity. But can capacity itself increase with motivation? Across four experiments $(\mathrm{N}=30-34)$ we demonstrate motivational improvements in WM even when all items were rewarded. However, this was not due to better memory precision, but rather better selection of the probed item within memory. Motivational improvements operated independently of encoding, maintenance, or attention shifts between items in memory. Moreover, motivation slowed responses. This contrasted with the benefits of rewarding items unequally, which allowed prioritisation of one item over another. We conclude that motivation can improve memory recall, not via precision or capacity, but via speed-accuracy trade-offs when selecting the item to retrieve.

\section{Statement of Significance}

Working memory has long been considered a fixed capacity resource, where remembering one item better always trades off with other items, which are remembered poorly. Motivation by reward can improve performance in a variety of domains, often breaking such trade-offs, giving a 'true improvement'. We investigated whether motivation by reward incentives could improve working memory without such prioritisation trade-offs, and reveal a 'true improvement'. Indeed people could improve their memory for multiple items when motivated, but not by increasing the encoding or storage precision. Instead, we found a remarkably simple mechanism behind this: motivation slowed target selection during retrieval to improve accuracy. This motivational strategy is explained by a cost of time, and contrasts with the prioritisation that occurred when items were unequally rewarded. Our findings reflect a flexible cache of motivational processes available to improve performance despite fixed limits in the WM system.

\section{Introduction}

Working memory (WM) is a short-term, flexible store for manipulating items in memory. There has been a wealth of research aimed at finding the limits of WM capacity, with most agreeing there is a fixed limit on the amount of information that can be stored, which can be spread between the items to be stored (Bays \& Husain, 2008; Burak \& Fiete, 2012; Miller, 1956; Schneegans \& Bays, 2016; van den Berg, Shin, Chou, George, \& Ma, 2012), although the exact limit is still unclear. A fixed limit seems at odds with our subjective experience of memory, where it feels like we can improve our 
performance when we are truly motivated to do so. Here we investigate whether people can actually improve WM when motivated, and what mechanisms might underlie this.

Recent research in other domains, such as decision making or motor precision, has found that people can improve their performance when motivated, and this can even occur without any associated downsides or trade-offs, suggesting a true benefit beyond the normal limits assumed (Codol, Holland, Manohar, \& Galea, 2019; Manohar, Muhammed, Fallon, \& Husain, 2019). There are costs associated with such boosted performance, including energetic costs of responding quicker, attentional demands, and opportunity costs as time spent processing one task is time not spent on other functions (Shenhav et al., 2017; Yee \& Braver, 2018). Rewards can offset these costs and motivate us to spend extra effort for the greater value outcomes on offer (Manohar et al., 2015).

Studies employing such incentivised motivation in WM have found that people can prioritise one item in WM (Hitch, Hu, Allen, \& Baddeley, 2018b; Hu, Allen, Baddeley, \& Hitch, 2016; Hu, Hitch, Baddeley, Zhang, \& Allen, 2014; Klink, Jeurissen, Theeuwes, Denys, \& Roelfsema, 2017). This incurs a cost of worse memory for the non-prioritised items, suggesting that there is a fixed WM capacity or resource which is strategically reallocated between items depending on their value and likelihood of being probed (Hitch et al., 2018b; Klink et al., 2017). Such prioritisation can also make items more susceptible to retroactive interference from a 'suffix' item (Hu et al., 2016), another form of tradeoff. This trade-off even operates between different modalities, where rewards for visual items improved visual WM capacity at the cost of auditory WM capacity on a concurrent task (Morey, Cowan, Morey, \& Rouder, 2011).

Prioritisation has usually been measured using a pre-stimulus cue (Hitch, Hu, Allen, \& Baddeley, 2018a; Hu et al., 2016), and comparing this against cues given after the stimuli (during maintenance or at retrieval) suggested prioritisation was only possible if the cues were present before (or possibly during) encoding (Klink et al., 2017). But encoding is not the only stage of WM, and it may be that maintenance and retrieval stages are open to modulation by reward. For instance, sustained activity and synaptic plasticity are both proposed to play a role during WM maintenance (Manohar, Zokaei, Fallon, Vogels, \& Husain, 2019; Schneegans \& Bays, 2018; Stokes, 2015) and are modifiable by dopamine (Shen, Flajolet, Greengard, \& Surmeier, 2008; Vijayraghavan, Wang, Birnbaum, Williams, \& Arnsten, 2007), which primarily signals reward and motivation (Berridge, 2007). WM retrieval, although relatively understudied, has been described by a decision process (Pearson, Raskevicius, Bays, Pertzov, \& Husain, 2014), where memory evidence is accumulated until a decision threshold is reached. To optimise reward, such decision processes ought to be under motivational control, since simple adjustments to the threshold induce speed-accuracy trade-offs, making responses either fast and inaccurate, or slow and accurate (Bogacz, Brown, Moehlis, Holmes, \& Cohen, 2006). The decision stages of WM are indeed flexible (Pearson et al., 2014) and presumably amenable to motivational control, so it is surprising that no such links have been found yet.

One explanation is that the reward manipulations used in the prioritisation tasks usually take the form of differential relative rewards, where some items are worth more than others, allowing strategic prioritisation of a fixed resource in order to maximise expected rewards. However, this will not always be the case in real life; sometimes we need to remember everything well, in which case improving performance for all items in memory would be favoured, if it were possible. The resource rational theory of WM (van den Berg \& Ma, 2018) suggests that rather than a fixed WM capacity, it is the costs of decreasing WM errors that limit performance, giving a mechanism for rewards to offset this cost and thus improve WM. A recent study from these authors failed to find such a rewardbased improvement (van den Berg, Zou, \& Ma, 2020), which may have been due to the betweensubject design and focus on precision rather than item selection during analysis. WM motivational 
processes could involve better encoding for all items (e.g. increased WM capacity), better maintenance over time (e.g. via reduced drift and decay of memories), or better retrieval of items (e.g. slower and more accurate decision processes). We aimed to see whether people can improve their WM when all items are highly rewarded (and thus prioritisation is not possible), and if so, what mechanisms are invoked.

We first measured the reward benefits on encoding, maintenance and retrieval phases of WM. We next replicated our findings with a greater focus on the encoding stage. Thirdly, we investigated whether reward may be affecting preparation of items for retrieval, by cueing an item during the delay. We then directly compared the motivational effects of equal rewards, where prioritisation is not possible, to unequal rewards, which allow prioritisation. Finally, we present data from control tasks without any WM demands to investigate how much of the reward effects can be attributed to the motor aspect of responses rather than WM per se.

\section{Experiment 1}

In the first experiment, we tested whether people could improve their WM accuracy when higher rewards were available, and which stage of WM this affected: encoding; maintenance; or retrieval.

\section{Methods}

\section{Participants}

We recruited 30 healthy young participants from the SONA website and tested them in the Anna Watts Building in the University of Oxford. We calculated an effect size of 30 to detect a medium effect of .5 with $\alpha=.05$ and $\beta=.84$. Participants gave written informed consent. Ethical approval for the study was granted by the University of Oxford Medical Sciences Interdivisional Research Ethics Committee (R45265/RE001).

Table 1. Participant demographics for all experiments.

\begin{tabular}{llll|} 
Experiment & $\mathrm{N}$ & Gender (M:F) & Mean Age (SD) \\
\hline 1 & 30 & $12: 17$ & $24.30(5.07)$ \\
\hline 2 & 34 & $9: 25$ & $24.53(5.77)$ \\
\hline 3 & 30 & $15: 15$ & $23.13(4.73)$ \\
\hline 4 & 30 & $10: 20$ & $23.07(4.72)$ \\
\hline $5 a$ & 72 & $22: 50$ & $23.65(5.33)$ \\
\hline $5 b$ & 15 & $10: 5$ & $23.87(4.91)$ \\
\hline
\end{tabular}

\section{Procedure}

We presented an array of randomly oriented coloured arrows and asked participants to reproduce the orientation of one arrow after a short delay by moving the mouse (Figure 1a; see Supplementary Materials for full details). Accurate responses were worth $1 p$ or $50 p$, which had been shown earlier in the trial. To investigate encoding, we presented the reward cue either before or after the stimuli. To investigate maintenance, a long or short delay was given after the post-stimuli coin/dot. Thus, there were three factors (high/low reward, pre/post cue, short/long delay), tested in a factorial design, giving eight conditions.

\section{Analysis}

All analyses were performed in Matlab R2018b (see Supplementary Materials for full details). We used repeated-measures ANOVA to analyse mean absolute angular error, and reaction time (RT). RTs were log-transformed for statistical testing (figures show raw RT). 
We also analysed the development of the response using the error of the mouse cursor trajectory. We interpolated 100 time-points between the start and end of the mouse movement, and used cluster permutation testing to see when the reward benefit appeared.

\section{Modelling}

To see what types of errors were affected by motivation, we fit mixture models to the angular errors. The misbinding model (Bays, Catalao, \& Husain, 2009) decomposes errors into three sources: imprecision (the width of the Von Mises distribution centred on the target orientation); misbinding (the proportion of responses centred around non-target locations); and random guesses (a uniform distribution spread over the entire circle of possible orientations). We fit this model to each condition separately, and report the mean parameters for the misbinding model (which fit the data better than a model without misbinding: $\Delta \mathrm{BIC}=-47.9$ ).

We also fit the models to each of the 100 interpolated time-points from the response trajectory, and compared the effect of high rewards with cluster-wise permutation tests.

We also fit a Linear Ballistic Accumulator (LBA) model (Brown \& Heathcote, 2008) to the data to characterise strategic changes in response time with reward (see Supplementary Materials for details).

Data Availability

All data and the experimental tasks are freely available on OSF (https://osf.io/tfnzw), and the analysis code is available on GitHub (https://doi.org/10.5281/zenodo.4421377).

\section{Results}

\section{Behaviour}

Participants had smaller absolute angular error when they were playing for $50 p$ than $1 p(p<.0001$, see Figure $1 \mathrm{c}$ and Table 2 for statistics), while they had larger errors when delays were long (vs short; $\mathrm{p}<.0001)$. There was no difference between trials with pre- or post-stimuli, although there was a cue-time * delay interaction $(p=.0116)$ as there were greater errors when short-delays following a post-cue than a pre-cue. However, there were no interactions between reward and cue-time or delay $(p>.05)$, meaning that reward had the same benefit whether it was given before or after the stimuli, and whether there was a short or long delay.

RT was significantly slowed by higher rewards ( $p<.0001$; Table 2 , Figure $1 d)$, with no other significant effects or interactions ( $p>.05$ ). This suggests people slowed their responses when motivated to perform better, which coincided with increased accuracy. But are people only more accurate because they are spending more time making their responses?

To answer this, we looked at accuracy during the response movement itself. We measured the absolute angular distance from the target orientation to the response orientation as a function of time during the movement. Permutation tests showed high rewards significantly lowered errors from the very start of the movement through until the end (Figure 1 e, cluster of $p<.05$ ). This means that people were not simply continuing their responses for longer to become more accurate when high rewards were available, but that they were already more accurate even when they started their response. 
a)

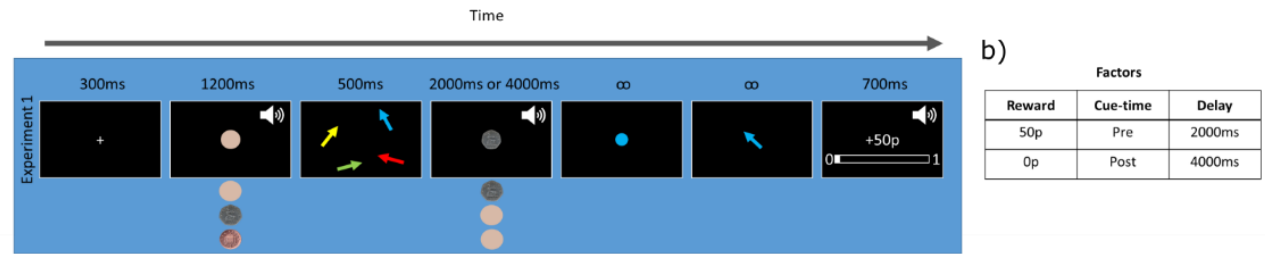

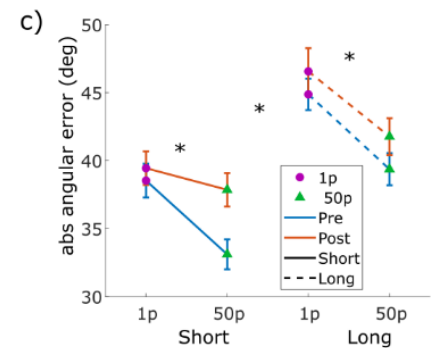

f)

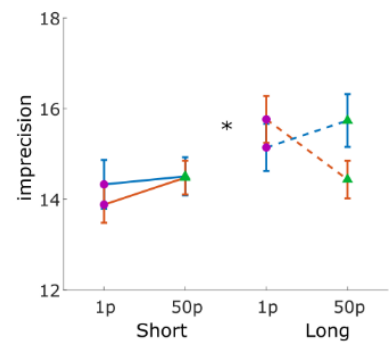

d) 1800

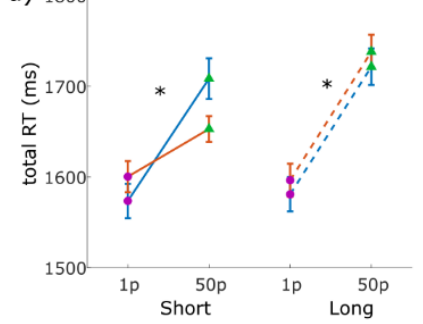

g)

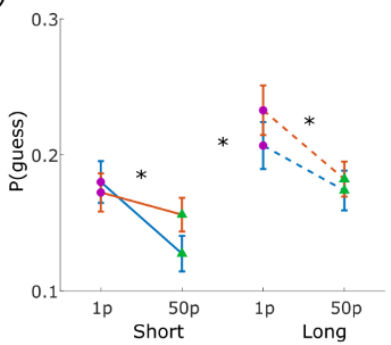

e) 48

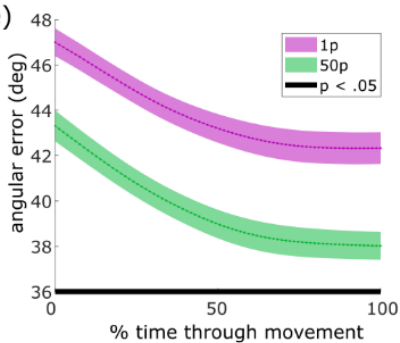

h)

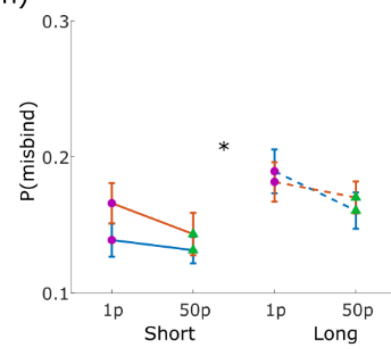

Figure 1. Reward improves WM accuracy via lower guessing and slower RT in Experiment 1. a) Trial structure for Experiment 1. A low/high reward was shown either before or after the four stimuli, and a beige placeholder dot was shown at the other time. After a short/long delay, one stimulus was probed by the colour of the dot, which became and arrow tracking the cursor when the participant began their response. After they clicked to give their response, a reward screen was shown, tracking their current and total rewards. b) Table showing the factors in the experiment: reward level, cue-time, and delay length, which were all tested in a factorial design giving eight different trial types. c) Higher rewards decreased angular error, independent of cue-time or delay length. d) Reward slowed RT, regardless of other factors. e) Reward decreased angular error from the start of the response movement (black bar $=p<.05$, cluster permutation tests). $f$ ) Imprecision of responses was not affected by reward. g) Reward decreased guessing, h) but did not significantly affect misbinding. All error bars show SEM.

Table 2. Statistical outputs from three-way repeated measure ANOVA (reward*cue-time*delay) on the behavioural and model parameters in Experiment 1. Significant effects are highlighted in red, $*=p<.05, * *=p<.01, * * *=p<.001$.

\begin{tabular}{|c|c|c|c|c|}
\hline Measure & Effect & $F(1,232)$ & p & $\eta_{p}^{2}$ \\
\hline \multirow[t]{7}{*}{ Error } & Reward & 20.4310 & $<.0001 * * *$ & 0.0809 \\
\hline & Cue-time & 0.16660 & 0.6835 & 0.0007 \\
\hline & Delay & 38.0339 & $<.0001 * * *$ & 0.1408 \\
\hline & Reward*Cue-time & 0.66170 & 0.4168 & 0.0028 \\
\hline & Reward*Delay & 0.73760 & 0.3913 & 0.0032 \\
\hline & Cue-time*Delay & 6.47220 & $0.0116^{*}$ & 0.0271 \\
\hline & Reward*Cue-time*Delay & 1.40870 & 0.2364 & 0.0060 \\
\hline \multirow[t]{2}{*}{ RT } & Reward & 94.8125 & $<.0001 * * *$ & 0.2901 \\
\hline & Cue-time & 0.02040 & 0.8867 & 0.0001 \\
\hline
\end{tabular}




\begin{tabular}{|c|c|c|c|c|}
\hline & Delay & 2.84360 & 0.0931 & 0.0121 \\
\hline & Reward*Cue-time & 1.82380 & 0.1782 & 0.0078 \\
\hline & Reward*Delay & 1.59880 & 0.2073 & 0.0068 \\
\hline & Cue-time*Delay & 0.00340 & 0.9537 & 0.0000 \\
\hline & Reward*Cue-time*Delay & 0.21610 & 0.6424 & 0.0009 \\
\hline \multirow[t]{7}{*}{ Imprecision } & Reward & 0.0007 & 0.9782 & 0.0000 \\
\hline & Cue-time & 0.0210 & 0.8849 & 0.0001 \\
\hline & Delay & 7.5292 & $0.0065^{* *}$ & 0.0314 \\
\hline & Reward*Cue-time & 2.7337 & 0.0996 & 0.0116 \\
\hline & Reward*Delay & 1.1323 & 0.2884 & 0.0049 \\
\hline & Cue-time*Delay & 0.6653 & 0.4155 & 0.0029 \\
\hline & Reward*Cue-time*Delay & 1.1297 & 0.2889 & 0.0048 \\
\hline \multirow[t]{7}{*}{$\mathbf{P}($ target $)$} & Reward & 24.8249 & $<.0001 * * *$ & 0.0967 \\
\hline & Cue-time & 0.2809 & 0.5966 & 0.0012 \\
\hline & Delay & 39.6085 & $<.0001^{* * *}$ & 0.1458 \\
\hline & Reward*Cue-time & 0.2266 & 0.6345 & 0.0010 \\
\hline & Reward*Delay & 0.3066 & 0.5803 & 0.0013 \\
\hline & Cue-time*Delay & 4.6094 & $0.0328 *$ & 0.0195 \\
\hline & Reward*Cue-time*Delay & 0.2180 & 0.6410 & 0.0009 \\
\hline \multirow[t]{7}{*}{ P(guessing) } & Reward & 11.9459 & $0.0007 * * *$ & 0.0490 \\
\hline & Cue-time & 0.0914 & 0.7627 & 0.0004 \\
\hline & Delay & 13.0963 & $0.0004 * * *$ & 0.0534 \\
\hline & Reward*Cue-time & 1.4937 & 0.2229 & 0.0064 \\
\hline & Reward*Delay & 0.1084 & 0.7423 & 0.0005 \\
\hline & Cue-time*Delay & 1.5710 & 0.2113 & 0.0067 \\
\hline & Reward*Cue-time*Delay & 0.1794 & 0.6723 & 0.0008 \\
\hline \multirow[t]{5}{*}{ P(misbinding) } & Reward & 3.0224 & 0.0834 & 0.0129 \\
\hline & Cue-time & 0.8335 & 0.3622 & 0.0036 \\
\hline & Delay & 9.0458 & $0.0029 * *$ & 0.0375 \\
\hline & Reward*Cue-time & 0.6452 & 0.4226 & 0.0028 \\
\hline & Reward*Delay & 0.0639 & 0.8006 & 0.0003 \\
\hline
\end{tabular}




$\begin{array}{llll}\text { Cue-time*Delay } & 1.0111 & 0.3157 & 0.0043 \\ \text { Reward*Cue-time*Delay } & 0.0030 & 0.9565 & 0.0000\end{array}$

\section{Mixture Modelling}

We fit the misbinding model to each condition separately to see whether reward was improving accuracy by decreasing imprecision, misbinding, or guessing. Rewards did not affect imprecision ( $p>$ .05 ; see Table 2 and Figure 1f) but increased target selection $(p<.0001)$ and decreased guessing (Figure 1g; $p<.0001$ ), without affecting misbinding (Figure $2 h ; p>.05$ ).

This pattern contrasted with the effect of delay, which increased imprecision $(p=.0065)$, and decreased target selection $(p<.0001)$ by increasing both random guessing $(p<.0001)$ and misbinding $(p=.0029)$. Importantly, there were no interactions between reward and delay or cuetime (although cue-time and delay did interact for target selection $(p=.0328)$ as delay had less effect if the cue was given after the stimuli). This all suggests that reward was decreasing error by increasing target selection, which was independent of cue-time or delay.

\section{Decision modelling}

Reward increased the threshold for the item-selection decision $(p=.0415$; see Supplementary Table S1 for full statistics), without affecting any other parameters ( $p>.05)$. In contrast, long delays decreased the drift rate for the target $(p<.0001)$ and the threshold $(p=.002)$. This suggests that when deciding which stimulus to select during retrieval, long delays can decrease the amount of information available, while rewards increase the decision threshold, making the decision more conservative by trading speed for accuracy.

\section{Experiment 2}

The lack of encoding benefit of motivation in Experiment 1 was surprising, as previous studies on prioritisation found that showing rewards before encoding gave greater benefits (Allen \& Ueno, 2018; Klink et al., 2017; Wallis, Stokes, Arnold, \& Nobre, 2015). We thus aimed to replicate the findings, but with greater emphasis on the encoding stage of the task.

\section{Methods}

We altered the task to make it easier for people to use motivational cues at encoding (Figure 2a; see Supplementary Materials). We used a longer reward-cue duration, and gave six blocks of pre-cued trials, and six blocks of post-cued trials (pseudorandomised block order) to remove any switching costs of cue-time. A single maintenance delay was used. On $12.5 \%$ of trials, after memory recall, we asked participants which reward they were playing for, to check they remembered this information ("catch trials"). Incorrect answers meant participants did not win any rewards even if their orientation response was accurate enough. There were 12 blocks of 32 trials, given in a pseudorandomised order, to 34 participants.

\section{Results}

\section{Behaviour}

Participants were quicker and more accurate to answer the catch trials when the reward was high ( $p$ $<.0001$; see Supplementary Materials), but catch trial accuracy did not affect angular error ( $p>.1)$, and moreover catch trials only differed from non-catch trials after memory recall, so we collapsed them together for subsequent analysis. 
Reward decreased mean absolute error (Figure 2c; Table 3; $p=.0016)$, as did pre-cues $(p=.0298)$, but there was no interaction $(p=.9766)$, replicating our Experiment 1 finding. RT was slowed by rewards as before (Figure $2 d$; Table $3 ; p<.0001)$, but also by post-cues $(p=.0260)$ and these interacted $(p=.0414)$ as the slowing was greater if pre-cued. The reward benefit on error was again seen right from the start of the response movement and persisted until the end (Figure 2e; $p<.05$ ), confirming that rewards do not improve accuracy only by slowing responses.

a)

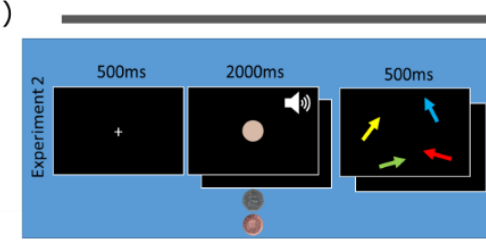

c)

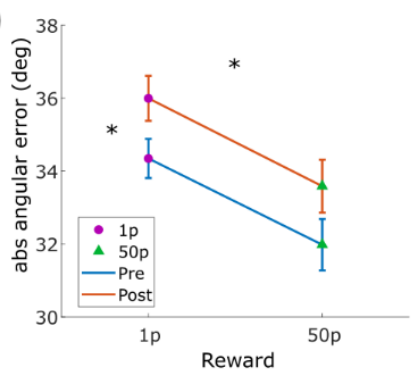

f)

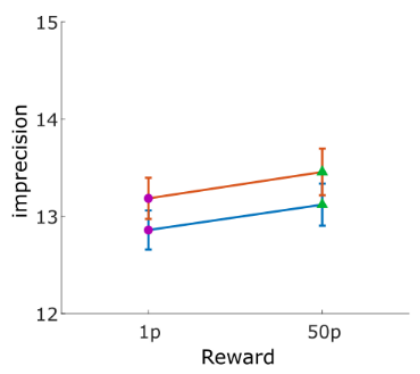

g)
Time
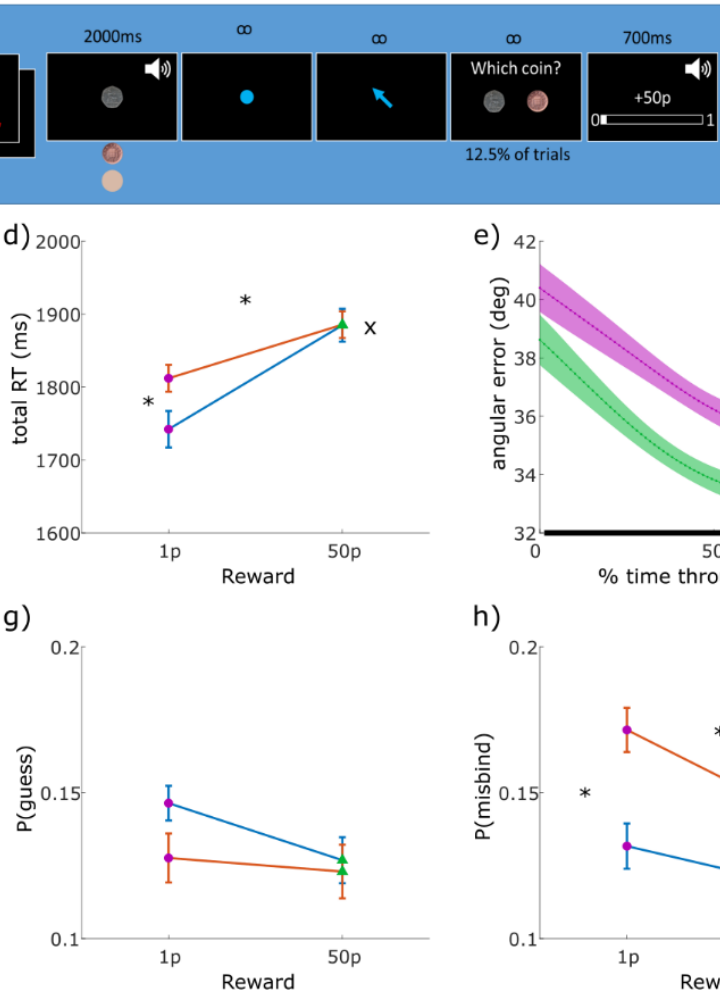

e) 42

b)
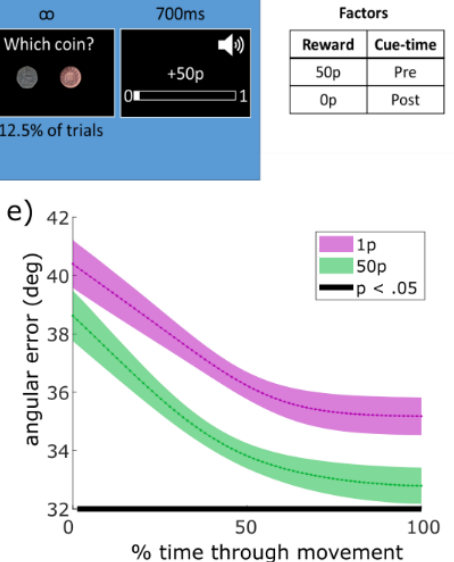

h)

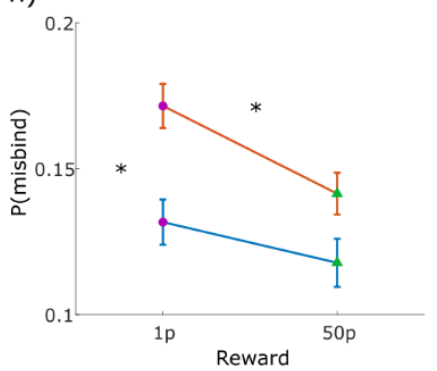

Figure 2. Reward improves WM accuracy via less misbinding, and slows RT in Experiment 2. a) Low or high rewards were shown before or after stimuli (beige dot shown at other time), with a fixed 2000ms delay period. On $12.5 \%$ of trials, after the response phase, participants were asked which reward they were playing for, with incorrect answers negating any rewards won for that trial. b) Factors for this experiment were reward level and cue-time. c) High rewards decreased mean absolute angular error for both pre-and post-stimuli cues. d) Rewards slowed RT, and this effect is greater for pre-cues. e) Response trajectories show reward significantly decreases errors across the whole movement. f) Reward did not affect imprecision. g) Reward did not affect guessing. h) Reward significantly decreased misbinding. All error bars show SEM.

Table 3. Statistical outputs from two-way repeated measure ANOVA (reward*cue-time) on the behavioural and model parameters in Experiment 2. Significant effects are highlighted in red, $*=p<.05, * *=p<.01, * * *=p<.001$.

\begin{tabular}{|c|c|c|c|c|}
\hline Measure & Effect & $F(1,132)$ & $\mathbf{p}$ & $\eta_{p}^{2}$ \\
\hline \multirow[t]{3}{*}{ Error } & Reward & 10.3940 & $0.0016 * *$ & 0.0730 \\
\hline & Cue-time & 4.8274 & $0.0298 *$ & 0.0353 \\
\hline & Reward*Cue-time & 0.0009 & 0.9766 & 0.0000 \\
\hline \multirow[t]{2}{*}{ RT } & Reward & 30.1080 & $<.0001 * * *$ & 0.1857 \\
\hline & Cue-time & 5.0706 & $0.0260 *$ & 0.0370 \\
\hline
\end{tabular}




\begin{tabular}{|c|c|c|c|c|}
\hline & Reward*Cue-time & 4.2428 & $0.0414^{*}$ & 0.0311 \\
\hline \multirow[t]{3}{*}{ Imprecision } & Reward & 1.1555 & 0.2844 & 0.0087 \\
\hline & Cue-time & 1.7831 & 0.1841 & 0.0133 \\
\hline & Reward*Cue-time & 0.0005 & 0.9830 & 0.0000 \\
\hline \multirow[t]{3}{*}{$\mathrm{P}$ (target) } & Reward & 15.1226 & $0.0002 * * *$ & 0.1028 \\
\hline & Cue-time & 5.4214 & $0.0214^{*}$ & 0.0395 \\
\hline & Reward*Cue-time & 0.0049 & 0.9444 & 0.0000 \\
\hline \multirow[t]{3}{*}{ P(guessing) } & Reward & 1.7984 & 0.1822 & 0.0134 \\
\hline & Cue-time & 1.5755 & 0.2116 & 0.0118 \\
\hline & Reward*Cue-time & 0.6810 & 0.4107 & 0.0051 \\
\hline \multirow[t]{3}{*}{$\mathbf{P}$ (misbinding) } & Reward & 6.3268 & $0.0131 *$ & 0.0457 \\
\hline & Cue-time & 13.1795 & $0.0004 * * *$ & 0.0908 \\
\hline & Reward*Cue-time & 0.8497 & 0.3583 & 0.0064 \\
\hline
\end{tabular}

Mixture Modelling

We fit the misbinding model to the four conditions. Imprecision was not affected by cue-time, reward or the interaction (Figure 2f; Table 3; $p>.05$ ). Rewards increased target responding ( $p<$ .0001 ) as in Experiment 1, but here decreased misbinding (Figure $2 h ; p=.0131$ ) rather than guessing (Figure $2 \mathrm{~g} ; \mathrm{p}=.1822)$. There were no interactions of reward and cue-time $(p>.05)$, although postcues did decrease target selection $(p=.0214)$ and increase misbinding $(p<.0001)$ without affecting guessing $(p>.05)$.

\section{Decision Modelling}

No parameters were significantly affected by rewards, cue-times or the interaction of the two ( $p>$ .05; see Supplementary Table S1).

\section{Experiment 3}

We wanted to find out what mechanisms could underlie the observed benefit of motivation on retrieval target selection, so Experiment 3 investigates whether motivation facilitates moving a selected item into the focus of attention (FoA). We asked participants to use one of the items in memory during the delay, independently of which item was probed at the end of the trial. This "incidental retrocue" brings one item into the FoA, improving subsequent recall for the cued item (Souza \& Oberauer, 2016). If reward facilitates shifts of attention, it should benefit items that were initially outside the FoA more than those already in the FoA, leading to interactions of reward and retrocue.

\section{Methods}

We presented the reward cue, then two arrows (Figure 3a; see Supplementary Materials), on the left and right side of the screen. During the delay, we printed the colour of one arrow on the screen and asked participants which side of the screen this arrow had appeared on, which they answered with a 
left/right mouse button click. This incidental retrocue was uninformative about which item had to be recalled at the end of the trial: on half the trials the cued arrow was probed (congruent trials) and on the other half the uncued arrow was probed (incongruent trials). There were 10 blocks of 32 trials, completed by 30 participants.

\section{Results}

\section{Behaviour}

The retrocue response accuracy and RT were not affected by reward $(p>.1)$, so we removed all trials where participants answered the retrocue incorrectly (1.29\% of trials overall). The findings did not change materially if we left these in, or if we excluded the one participant who scored below $95 \%$ on the retrocue.

As expected, congruent retrocues decreased memory error (Figure 3c; Table 4; $p<.0001$ ), as did high reward $(p=.0216)$, but importantly there was no interaction $(p=.9394)$, suggesting that rewards had the same benefit for items in or out of the FoA.

RT was sped up by congruent retrocues (Figure 3d; $p=.0083)$, while reward slowed RT $(p<.0001)$, with no significant interaction ( $p=.8017)$.

Again, rewards improved accuracy from the very start of the response trajectories until the end of movements (Figure $3 e ; p<.05$ ), showing the reward benefit was present at movement initiation.

a)

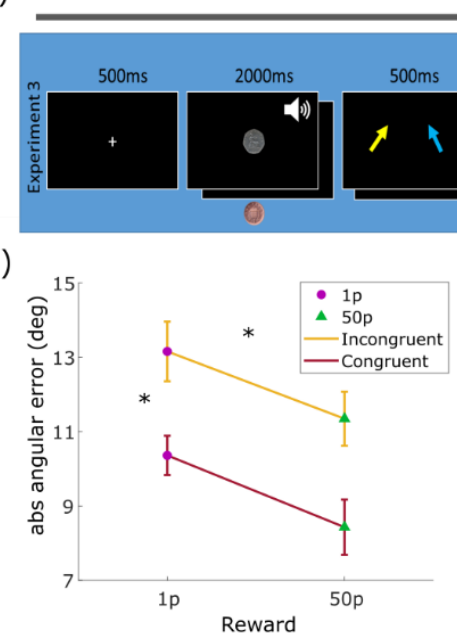

f)

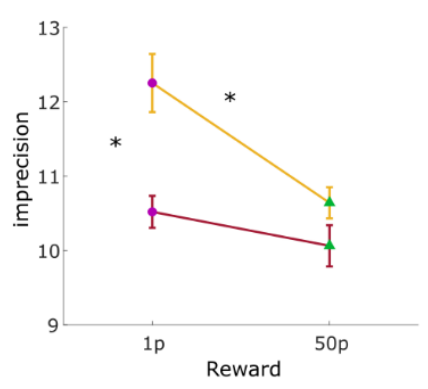

Tim

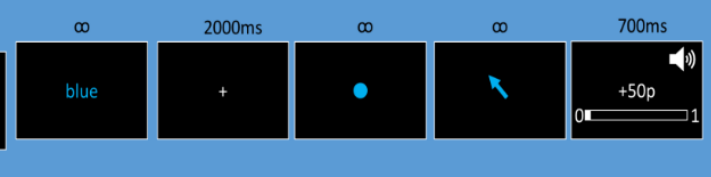

d)

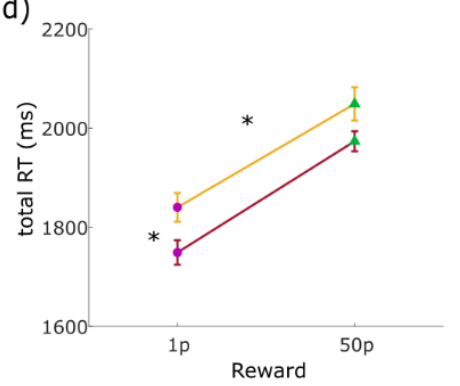

g) 0.06

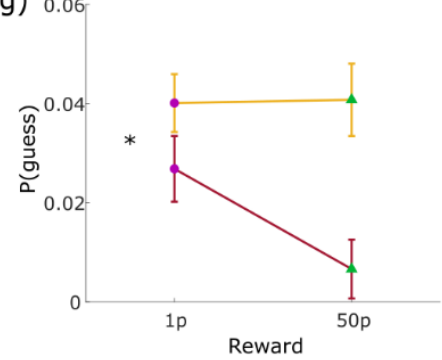

b)

e)
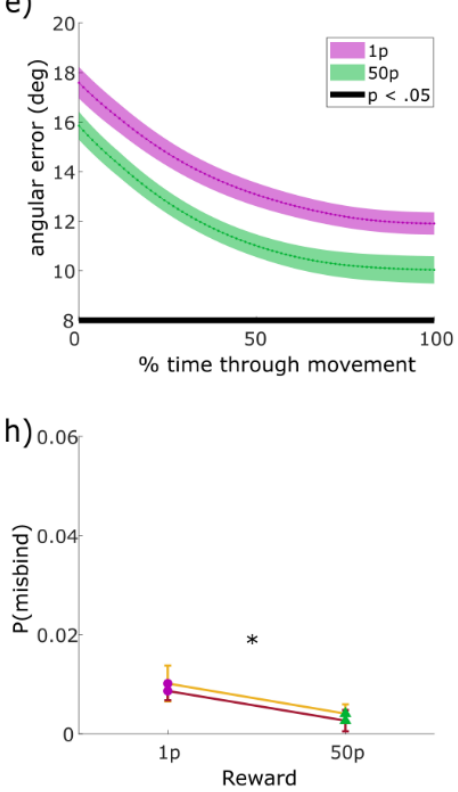

Figure 3. Reward affected congruently and incongruently cued items equally in Experiment 3. a) Trial structure: a high or low reward is shown before the two stimuli, then the colour of one stimulus is written and the participant must decide if that coloured arrow was on the left or right of the screen (mouse click response). This is the incidental retrocue, which activates that coloured arrow in WM. After a fixed delay, either the cued arrow is probed (congruent trials) or the uncued arrow (incongruent trials), and participants respond. b) The experimental factors are reward level and congruency of the incidental retrocue. c) High rewards decreased mean absolute angular error equally for congruent and incongruent items. 
d) Congruent retrocues sped up RT, but reward slowed RT. e) Response trajectories show smaller errors from the start of the movement. f) Rewards decreased imprecision similarly for congruent and incongruent retrocues. g) Rewards did not significantly affect guessing. h) Rewards decreased misbinding. All error bars show SEM.

Table 4. Statistical outputs from two-way repeated measure ANOVA (reward*congruency) on the behavioural and model parameters in Experiment 3. Significant effects are highlighted in red, $*=p<.05, * *=p<.01, * * *=p<.001$.

\begin{tabular}{|c|c|c|c|c|}
\hline Measure & Effect & $F(1,116)$ & p & $\eta_{p}^{2}$ \\
\hline \multirow[t]{3}{*}{ Error } & Reward & 5.4222 & $0.0216^{*}$ & 0.0447 \\
\hline & Congruency & 12.6398 & $0.0005 * * *$ & 0.0983 \\
\hline & Reward*Congruency & 0.0058 & 0.9394 & 0.0000 \\
\hline \multirow[t]{3}{*}{ RT } & Reward & 48.8467 & $<.0001 * * *$ & 0.2963 \\
\hline & Congruency & 7.2176 & $0.0083^{* *}$ & 0.0586 \\
\hline & Reward*Congruency & 0.0634 & 0.8017 & 0.0005 \\
\hline \multirow[t]{3}{*}{ Imprecision } & Reward & 10.3599 & $0.0017 * *$ & 0.0820 \\
\hline & Congruency & 12.9361 & $0.0005^{* * *}$ & 0.1003 \\
\hline & Reward*Congruency & 3.2242 & 0.0752 & 0.0270 \\
\hline \multirow[t]{3}{*}{ P(target) } & Reward & 3.4604 & 0.0654 & 0.0290 \\
\hline & Congruency & 8.7585 & $0.0037 * *$ & 0.0702 \\
\hline & Reward*Congruency & 1.4922 & 0.2244 & 0.0127 \\
\hline \multirow[t]{3}{*}{ P(guessing) } & Reward & 1.7824 & 0.1844 & 0.0151 \\
\hline & Congruency & 10.4706 & $0.0016 * *$ & 0.0828 \\
\hline & Reward*Congruency & 2.0289 & 0.1570 & 0.0172 \\
\hline \multirow[t]{3}{*}{ P(misbinding) } & Reward & 4.5549 & $0.0349 *$ & 0.0378 \\
\hline & Congruency & 0.2666 & 0.6066 & 0.0023 \\
\hline & Reward*Congruency & 0.0003 & 0.9867 & 0.0000 \\
\hline
\end{tabular}

Mixture Modelling

In contrast to the previous experiments, rewards decreased imprecision (Figure 3f; Table 4; $p=$ $.0017)$, as did congruent retrocues ( $p<.0001)$, but there was no significant interaction $(p=.0752)$. Rewards also decreased misbinding (Figure 3Figure $4 \mathrm{~h} ; \mathrm{p}=.0349$ ), but did not significantly affect guessing (Figure $3 g, p=.1844)$ or target selection $(p=.0653)$.

Decision Modelling

Reward did not affect any parameter, while congruent retro-cues increased the target drift rate $(p=$ .0013; see Supplementary Table S1). 


\section{Experiment 4}

As we have found no interactions of the motivational effect with encoding, maintenance, or focusing attention, which contrasts with previous findings of reward prioritisation, we aimed to replicate previous findings that rewarding one items leads to its prioritisation in WM, and directly contrasted this with the motivational strategy found in experiments 1-3. Specifically, previous studies have found that when one item is rewarded more than others, it is prioritised, giving higher accuracy for that item at the cost of lower accuracy for the others, and this effect is greater when the cues are shown before the stimuli, and offers protection from imprecision and guessing (Allen \& Ueno, 2018; Klink et al., 2017).

\section{Methods}

We showed participants two coloured arrows, each of which was worth a separate reward amount if remembered accurately when probed (Figure 4a; see Supplementary Materials). The reward cues were given either pre- or post-stimuli, in the same position as the associated arrow, and a fixed delay was used. Thirty participants completed 10 blocks of 48 trials.

\section{Results}

\section{Behaviour}

We first used a three-way repeated measures ANOVA, followed up by two two-way ANOVAs to compare equal-reward and unequal-reward trials separately when there was an interaction with equality.

High rewards for the probed item decreased mean angular error (Figure 4c; Table 5; $<<.0001$ ), and this effect was greater when the rewards were unequal (reward*equal interaction: $p<.0001$ ), indicating that prioritisation conferred greater benefits than motivation.

The separate ANOVAs showed that when both stimuli had equal rewards, high reward decreased error (Table 6; $p=.0051)$, while post-cues increased error $(p<.0001)$ with no interaction $(p=.2337)$. This replicates the findings from the previous experiments. When rewards were unequal, high rewards gave a much larger decrease in error (Table 6; $p<.0001$ ), with non-significant trends for post-cues increasing error $(p=.0606)$ and reward cues being more effective before encoding (reward*cue-time: $p=.0635$ ). 
a)

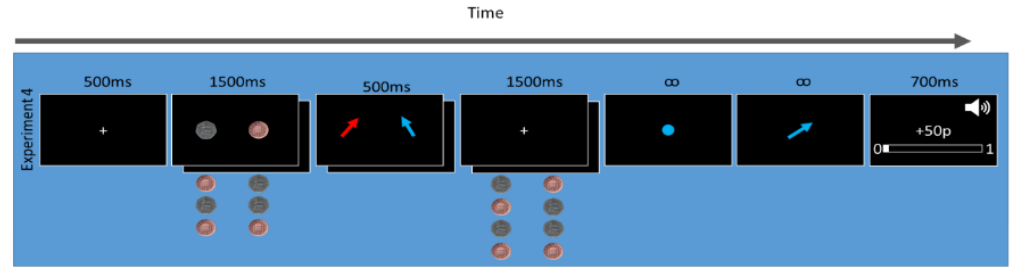

b)

c)

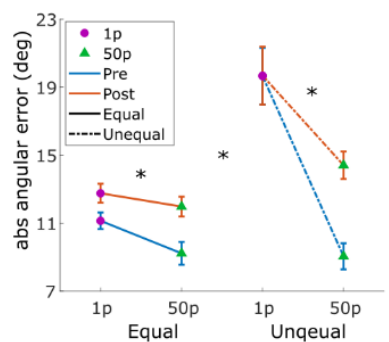

d) 1900

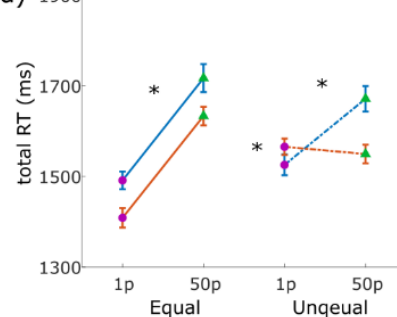

e)

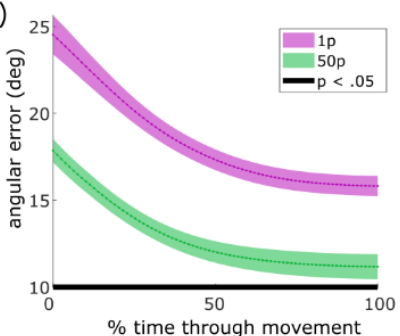

g) 0.15
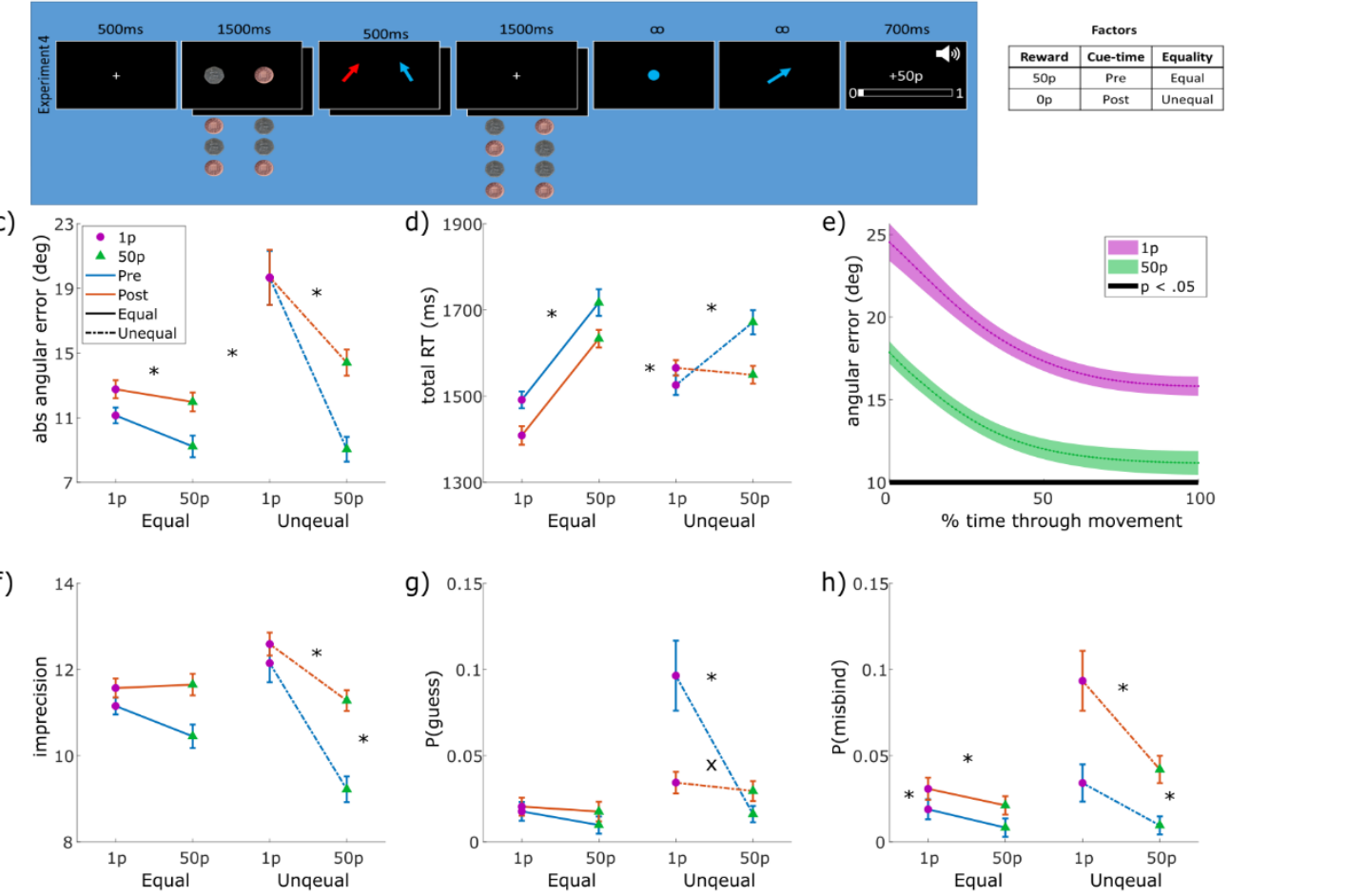

$\%$ time through movement

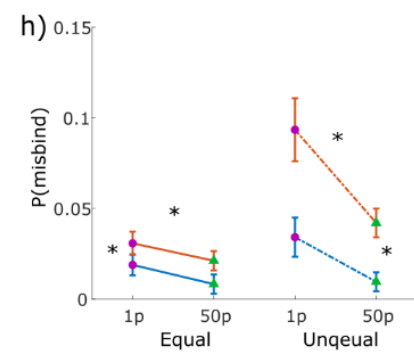

Figure 4. Reward affects WM differently when prioritisation is possible (Unequal) or not (Equal). a) Trial structure: rewards were shown for each stimulus, and could appear before or after the stimuli. The rewards were equal or unequal, and each could be high or low. One stimulus was probed, and that stimulus' reward was given if correct. b) The factors were reward, cue-time and equality of rewards. c) Rewards decreased mean absolute angular error, especially unequal rewards. d) Rewards slowed RT, as did pre-cues, and these interact only for the unequal rewards; when cued after stimuli, unequal rewards did not differ in RT as even 1p items were slowed. e) Response trajectories show reward decreases error across the whole movement. f) Imprecision is decreased by unequal reward, but unaffected by equal reward. g) Guessing was decreased by unequal rewards, but not equal rewards. When unequal rewards are cued before the stimuli, and people are probed on the low-reward item, they have high guessing rates. h) Equal and unequal rewards decreased misbinding, with the greatest effect for post-cued unequal rewards. All error bars shown SEM.

RT was again slowed by reward (Figure 4d; Table 5; $<<.0001$ ), and there was an interaction of reward*equality $(p<.0001)$. When rewards were equal between stimuli, high rewards slowed RT as in previous experiments (Table 6; $p<.0001$ ), and post-cues sped RT $(p=.0016)$, with no interaction. However, when rewards were unequal, responses to the high-reward stimulus were slower (Table 6; $p=.0097)$ while cue-time did not have a significant effect $(p=.0991)$, and these interacted $(p=$ .0014). People were slow to respond when unequal rewards had been presented after the stimuli, for both low- and high-rewarded items.

Response trajectories were more accurate from the start of movement when probed on a highreward target (Figure $4 \mathrm{e} ; \mathrm{p}<.05$ ), indicating the reward benefit was present when responses started.

Table 5. Statistical outputs from three-way repeated measure ANOVA (reward*cue-time*equality) on the behavioural and model parameters in Experiment 4. Significant effects are highlighted in red, ${ }^{*}=p<.05,{ }^{*}=p<.01,{ }^{* * *}=p<.001$.

\begin{tabular}{llcll} 
Measure & Effect & $\mathbf{F ~ ( 1 , ~ 2 3 2 )}$ & $\mathbf{P}$ & $\eta_{p}^{2}$ \\
\hline Error & Reward & 37.8151 & $<.0001^{* * *}$ & 0.1402 \\
& Cue-time & 10.4388 & $0.0014^{* *}$ & 0.0431
\end{tabular}




\begin{tabular}{|c|c|c|c|c|}
\hline & Equality & 34.3512 & $<.0001 * * *$ & 0.1290 \\
\hline & Reward*Cue-time & 4.5717 & $0.0335^{*}$ & 0.0193 \\
\hline & Reward*Equality & 19.0332 & $<.0001 * * *$ & 0.0758 \\
\hline & Cue-time*Equality & 0.1130 & 0.7371 & 0.0005 \\
\hline & Reward*Cue-time*Equality & 1.9282 & 0.1663 & 0.0082 \\
\hline \multirow[t]{7}{*}{ RT } & Reward & 34.2258 & $<.0001 * * *$ & 0.1286 \\
\hline & Cue-time & 0.0000 & 0.9984 & 0.0000 \\
\hline & Equality & 1.6763 & 0.1967 & 0.0072 \\
\hline & Reward*Cue-time & 0.0969 & 0.7558 & 0.0004 \\
\hline & Reward*Equality & 85.2225 & $<.0001 * * *$ & 0.2687 \\
\hline & Cue-time*Equality & 0.1216 & 0.7276 & 0.0005 \\
\hline & Reward*Cue-time*Equality & 0.2002 & 0.6550 & 0.0009 \\
\hline \multirow[t]{7}{*}{ Imprecision } & Reward & 33.5485 & $<.0001 * * *$ & 0.1263 \\
\hline & Cue-time & 24.0678 & $<.0001^{* * *}$ & 0.0940 \\
\hline & Equality & 0.2440 & 0.6218 & 0.0011 \\
\hline & Reward*Cue-time & 8.1586 & $0.0047^{* *}$ & 0.0340 \\
\hline & Reward*Equality & 18.5405 & $<.0001 * * *$ & 0.0740 \\
\hline & Cue-time*Equality & 1.1081 & 0.2936 & 0.0048 \\
\hline & Reward*Cue-time*Equality & 0.9818 & 0.3228 & 0.0042 \\
\hline \multirow[t]{7}{*}{$\mathbf{P}$ (target) } & Reward & 25.6868 & $<.0001 * * *$ & 0.0997 \\
\hline & Cue-time & 4.2917 & $0.0394 *$ & 0.0182 \\
\hline & Equality & 30.9882 & $<.0001 * * *$ & 0.1178 \\
\hline & Reward*Cue-time & 2.0795 & 0.1506 & 0.0089 \\
\hline & Reward*Equality & 11.7931 & $0.0007 * * *$ & 0.0484 \\
\hline & Cue-time*Equality & 0.0409 & 0.8398 & 0.0002 \\
\hline & Reward*Cue-time*Equality & 1.2599 & 0.2628 & 0.0054 \\
\hline \multirow[t]{5}{*}{ P(guessing) } & Reward & 13.4107 & $0.0003 * * *$ & 0.0546 \\
\hline & Cue-time & 2.1092 & 0.1478 & 0.0090 \\
\hline & Equality & 17.9654 & $<.0001 * * *$ & 0.0719 \\
\hline & Reward*Cue-time & 9.4393 & $0.0024 * *$ & 0.0391 \\
\hline & Reward*Equality & 8.0730 & $0.0049 * *$ & 0.0336 \\
\hline
\end{tabular}




\begin{tabular}{|c|c|c|c|c|}
\hline & Cue-time*Equality & 5.1213 & $0.0246 *$ & 0.0216 \\
\hline & Reward*Cue-time*Equality & 7.2156 & $0.0077 * *$ & 0.0302 \\
\hline \multirow[t]{7}{*}{$\mathbf{P}$ (misbinding) } & Reward & 13.0823 & $0.0004 * * *$ & 0.0534 \\
\hline & Cue-time & 19.1844 & $<.0001 * * *$ & 0.0764 \\
\hline & Equality & 14.1367 & $0.0002 * * *$ & 0.0574 \\
\hline & Reward*Cue-time & 0.9379 & 0.3338 & 0.0040 \\
\hline & Reward*Equality & 4.3958 & $0.0371^{*}$ & 0.0186 \\
\hline & Cue-time*Equality & 6.3240 & $0.0126 *$ & 0.0265 \\
\hline & Reward*Cue-time*Equality & 1.0896 & 0.2977 & 0.0047 \\
\hline
\end{tabular}

Table 6. Statistical outputs from separate two-way repeated measure ANOVA (reward*cue-time) on equal and unequal trials in the behavioural and model parameters in Experiment 4. Significant effects are highlighted in red, $*=p<.05, * *=p$ $<.01, * * *=p<.001$.

\begin{tabular}{|c|c|c|c|c|c|}
\hline Measure & Equality & Effect & $F(1,116)$ & p & $\eta_{p}^{2}$ \\
\hline \multirow[t]{6}{*}{ Error } & \multirow[t]{3}{*}{ Equal } & Reward & 8.1434 & $0.0051 * *$ & 0.0656 \\
\hline & & Cue-time & 21.3768 & $<.0001 * * *$ & 0.1556 \\
\hline & & Reward*Cue-time & 1.4332 & 0.2337 & 0.0122 \\
\hline & \multirow[t]{3}{*}{ Unequal } & Reward & 31.1821 & $<.0001 * * *$ & 0.2119 \\
\hline & & Cue-time & 3.5904 & 0.0606 & 0.0300 \\
\hline & & Reward*Cue-time & 3.5097 & 0.0635 & 0.0294 \\
\hline \multirow[t]{6}{*}{ RT } & \multirow[t]{3}{*}{ Equal } & Reward & 76.4571 & $<.0001 * * *$ & 0.3973 \\
\hline & & Cue-time & 10.4301 & $0.0016 * *$ & 0.0825 \\
\hline & & Reward*Cue-time & 0.0003 & 0.9858 & 0.0000 \\
\hline & \multirow[t]{3}{*}{ Unequal } & Reward & 6.9087 & $0.0097 * *$ & 0.0562 \\
\hline & & Cue-time & 2.7640 & 0.0991 & 0.0233 \\
\hline & & Reward*Cue-time & 10.7343 & $0.0014^{* *}$ & 0.0847 \\
\hline \multirow[t]{6}{*}{ Imprecision } & \multirow[t]{3}{*}{ Equal } & Reward & 1.6384 & 0.2031 & 0.0139 \\
\hline & & Cue-time & 11.0127 & $0.0012^{* *}$ & 0.0867 \\
\hline & & Reward*Cue-time & 2.5811 & 0.1109 & 0.0218 \\
\hline & \multirow[t]{3}{*}{ Unequal } & Reward & 37.1981 & $<.0001 * * *$ & 0.2428 \\
\hline & & Cue-time & 12.9518 & $0.0005 * * *$ & 0.1004 \\
\hline & & Reward*Cue-time & 5.3994 & $0.0219 *$ & 0.0445 \\
\hline
\end{tabular}




\begin{tabular}{|c|c|c|c|c|c|}
\hline \multirow[t]{6}{*}{$\mathrm{P}($ target $)$} & \multirow[t]{3}{*}{ Equal } & Reward & 5.8292 & $0.0173^{*}$ & 0.0478 \\
\hline & & Cue-time & 7.6278 & $0.0067^{* *}$ & 0.0617 \\
\hline & & Reward*Cue-time & 0.2230 & 0.6377 & 0.0019 \\
\hline & \multirow[t]{3}{*}{ Unequal } & Reward & 21.5777 & $<.0001 * * *$ & 0.1568 \\
\hline & & Cue-time & 1.5435 & 0.2166 & 0.0131 \\
\hline & & Reward*Cue-time & 1.9631 & 0.1639 & 0.0166 \\
\hline \multirow[t]{6}{*}{ P(guessing) } & \multirow[t]{3}{*}{ Equal } & Reward & 1.2840 & 0.2595 & 0.0109 \\
\hline & & Cue-time & 1.2527 & 0.2653 & 0.0107 \\
\hline & & Reward*Cue-time & 0.2842 & 0.5950 & 0.0024 \\
\hline & \multirow[t]{3}{*}{ Unequal } & Reward & 12.1227 & $0.0007 * * *$ & 0.0946 \\
\hline & & Cue-time & 3.9566 & $0.0490 *$ & 0.0330 \\
\hline & & Reward*Cue-time & 9.5049 & $0.0025^{* *}$ & 0.0757 \\
\hline \multirow[t]{6}{*}{ P(misbinding) } & \multirow[t]{3}{*}{ Equal } & Reward & 5.1785 & $0.0247 *$ & 0.0427 \\
\hline & & Cue-time & 7.7946 & $0.0061 * *$ & 0.0630 \\
\hline & & Reward*Cue-time & 0.0127 & 0.9104 & 0.0001 \\
\hline & \multirow[t]{3}{*}{ Unequal } & Reward & 10.2701 & $0.0017^{* *}$ & 0.0813 \\
\hline & & Cue-time & 14.9555 & $0.0002 * * *$ & 0.1142 \\
\hline & & Reward*Cue-time & 1.2739 & 0.2613 & 0.0109 \\
\hline
\end{tabular}

Mixture Modelling

Reward decreased imprecision (Figure 4f; Table 5; $p<.0001$ ), but this interacted with equality $(p<$ $.0001)$. Separate two-way ANOVA showed this was driven by prioritisation only, with reward decreasing imprecision when the items were unequal (Table 6; $p<.0001$ ) but not when they were equal $(p=.2031)$. Unequal rewards also had a greater benefit on precision when pre-cued (reward*cue-time: $p=.0219$ ).

Reward increased target selection (Table $5 ; p<.0001)$, which interacted with equality $(p<.0001)$. This was due to larger reward benefits when rewards were unequal (Table $6 ; p<.0001$ ) than equal $(p=.0173)$.

Guessing was decreased by reward (Figure 4g; Table 6; $p<.0001$ ), which interacted with equality ( $p$ $=.0049$ ). Separate ANOVAs showed that high reward decreased guesses only when items were unequal (Table $6 ; p<.0001)$ but not when they were equal $(p=.2595)$. In other words, participants guessed less only when asked about a prioritised item. This unequal reward benefit on guessing was greatest when the rewards were presented before the stimuli (reward*cue: $p=.0026$ ), which was driven by very high guessing for pre-cued low-reward stimuli, perhaps reflecting lack of encoding for this low value item. 
Misbinding was also decreased by reward (Figure 4h; Table $5 ; p<.0001$ ), and there was an interaction of reward*equality $(p=.0371)$. When rewards were equal, pre-cues (Table $6 ; p=.0247$ ) and high rewards $(p=.0061)$ both decreased misbinding. When rewards were unequal, these effects were much larger $(p=.0017 ; p<.0001)$. As there were only two stimuli in this experiment, misbinding means they reported the item with the other reward value - so more misbinding when probed on the low-reward item means participants were reporting the high-reward item more, especially when unequal rewards were given. In addition, when unequal rewards were shown after encoding, misbinding was higher for both reward values, suggesting that whatever prioritisation process occurred during the maintenance period (e.g. refocusing of attention, removal of an item from WM) may have also increased misbinding of features between them.

\section{Decision Modelling}

Target drift was increased by reward ( $p<.001$; see Supplementary Table S1 for full statistics), with no interaction with equality $(p>.05)$. Threshold was not affected by reward $(p=.1980)$, although there was a reward*equality interaction $(p=.0092)$, as equal rewards increased the threshold $(p=$ $.0062)$, while unequal high rewards did not $(p=.3570)$.

\section{Experiment 5a}

The majority of the motivational effects reported above coincided with a speed-accuracy trade-off, which potentially could result from people taking longer to fine-tune their final orientation response, in the absence of memory benefits. We have already offered evidence against this, such as the modelling showing reward benefits target selection rather than precision, and the benefit being present from the beginning of the response. However, to test this more directly, we included two motor control experiments where the response was the same but there were no WM demands.

\section{Methods}

We used a version of the previous task without any memory demands; one arrow was used and it stayed on the screen during the probe and response phases (Figure 5a; see Supplementary Materials). There were 24 trials, 12 for each reward, and this task was run before several of the above experiments, giving 72 participants.

We did not perform mixture modelling as there were no distractors to misbind to, and LBA modelling was not possible since there would be too few trials classed as errors.

\section{Results}

There were large practice effects with a steep drop in error and RT over the first two trials, so we excluded these two trials. Reward decreased error (Table 7; $p=.0180$ ) and slowed RT $(p<.0001)$. This reduction in error was only seen towards the end of the response movement trajectory (Figure $5 c ; p<.05)$, in contrast to the WM experiments. 
a)

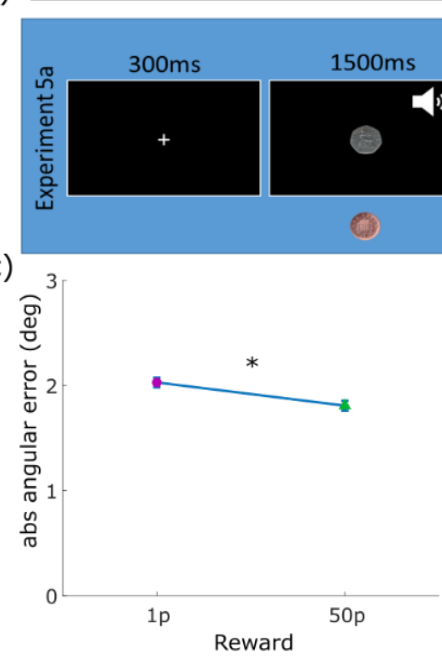

Time
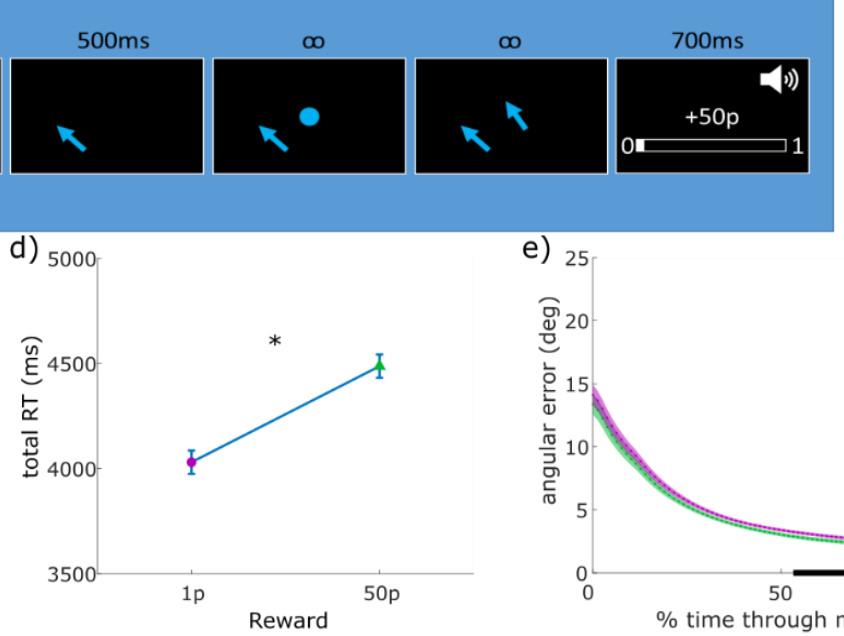

e) 25

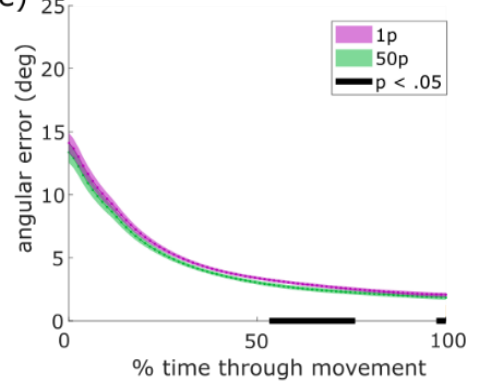

b)

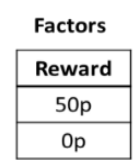

Time

f)

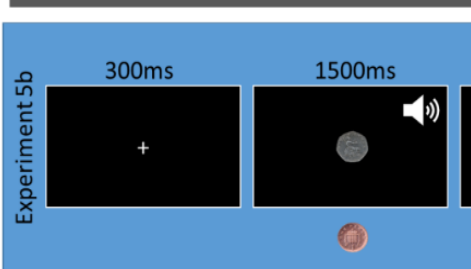

h)

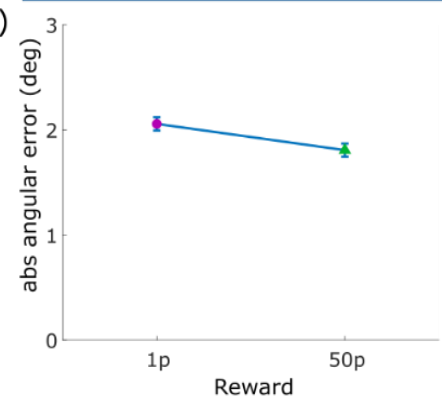

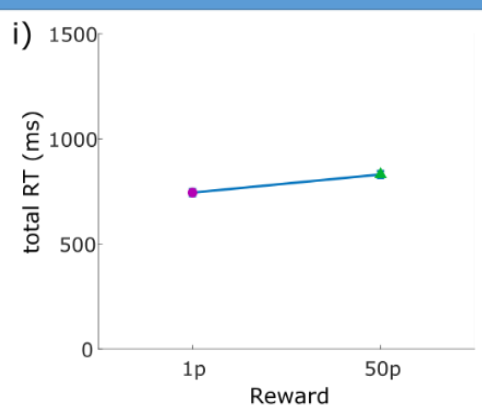

g)
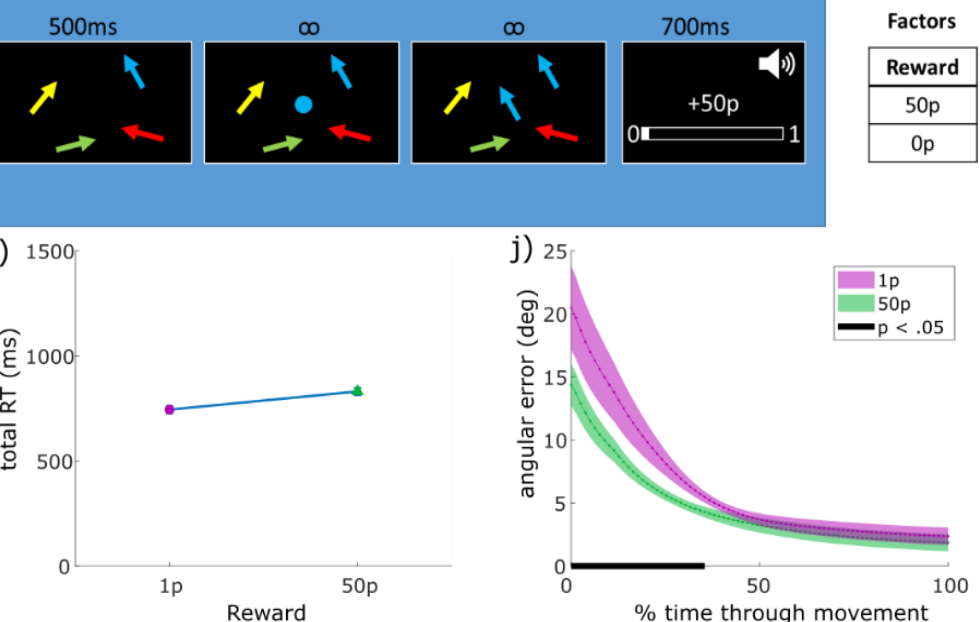

Figure 5. Reward decreases error and increases RT even in the absence of WM demands in Experiments $5 a$ \& $5 b$. Experiment 5a: a-e. a) Trial structure: low/high reward was shown before the stimulus, and the stimulus remained on screen during response, removing the need for WM. b) Reward was the only factor. c) Reward decreased error (after excluding first two trials). d) Reward slowed RT. e) Response trajectories show reward only decreased error towards the end of the response. Experiment 5b: $f$-j. f) Trial structure: Reward was shown before the stimuli, which remained on screen during response to remove the need for WM. g) Reward was the only factor. h) Reward did not significantly decrease error. i) Reward did not significantly slow RT. j) Reward decreased errors at the start of responses, but there was no significant difference by the end of the movements.

Table 7. Statistical outputs from one-way repeated measure ANOVA (reward) on the behavioural and model parameters in Experiments $5 a$ and $5 b$. We excluded the first two trials in experiment $5 a$ as there were large practice effects on those. Significant effects are highlighted in red, * $=p<.05, * *=p<.01, * * *=p<.001$.

\begin{tabular}{llllll} 
Experiment & Measure & Effect & $\mathbf{F}$ & $\mathbf{p}$ & $\eta_{p}^{2}$ \\
\hline $\mathbf{5 a}(\mathbf{d f}=\mathbf{1 , \mathbf { 1 4 2 }})$ & Error & Reward & 5.7281 & $0.0180^{*}$ & 0.0388 \\
& RT & Reward & 11.6924 & $0.0008^{* * *}$ & 0.0761 \\
$\mathbf{5 b}(\mathbf{d f}=\mathbf{1 , 2 8})$ & Error & Reward & $\mathbf{2 . 5 9 8 8}$ & 0.1182 & 0.0849
\end{tabular}




\section{Experiment $5 b$}

\section{Methods}

As the previous data showed large practice effects and was fairly noisy due to low trial numbers, we modified the task to have more trials and to more closely match the memory tasks, only without the memory component. Four stimuli were shown (Figure 5f; see Supplementary Materials), and remained on the screen during probe and response, and there were six practice trials before two blocks of 30 trials, completed by 15 participants.

\section{Results}

Reward did not significantly decrease error (Figure $5 d$; Table 7; $p=.1181$ ) or affect RT (Figure $5 e ; p=$ .1827). There was a reward benefit early on in the response trajectory, but this did not persist until the end of the trial $(p>.05)$.

\section{Correlations}

As a further test, we compared the effect of reward on error and RT in the motor and WM experiments. If the WM accuracy benefit of reward is due to more time spent fine-tuning the motor response (which is present in both motor and WM tasks), then there should be correlations between the reward effects on error and RT in the motor task and the WM task. We regressed the motor reward effects for the 84 participants who completed either version of the motor task, with the reward effects from whichever WM experiment they completed (Experiment 2, 3 or 4).

There was a significant correlation between motor and WM RT (Figure 6; $r^{2}=.1298, F=24.1632, p<$ $.0001)$, but there was no significant association between motor and WM errors $\left(r^{2}=.0187, F=\right.$ $3.0850, p=.0809)$.
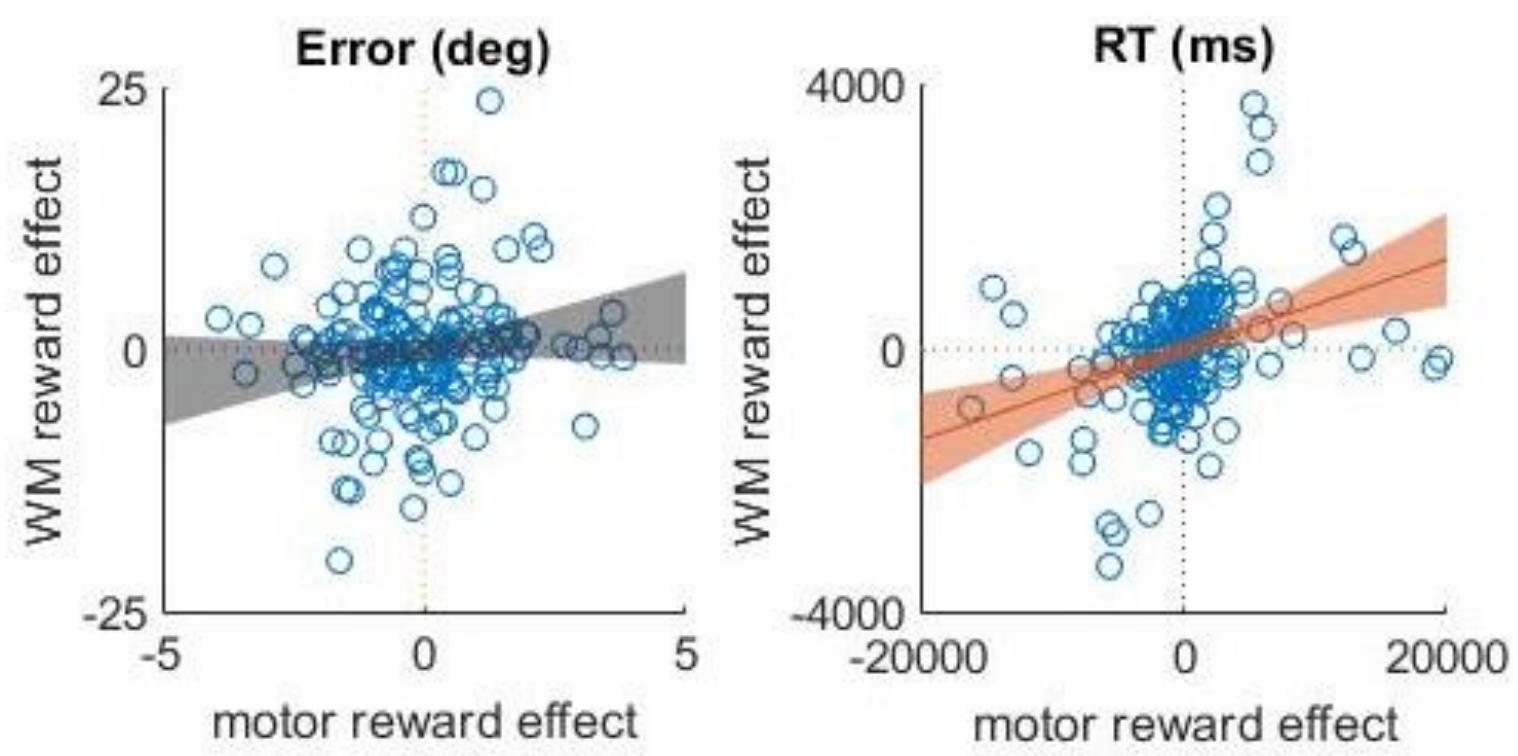

Figure 6. WM RT, but not error, is association with motor task responses. We regressed WM reward effects (difference between 50p and $1 p$ conditions across Experiments 2-4) with motor reward effects (50p-1p for Experiments 5a\&b) in the same people. a) Motor and WM errors were not associated ( $p=.0809)$, while $b)$ RT was significantly associated between 
experiment types $(p<.0001)$. Orange lines are significant regression lines with 95\% confidence intervals shaded, while grey shading is $95 \%$ confidence intervals on non-significant regression line which include $y=0$ line.

\section{Across-experiment trajectory modelling}

While the motor-WM correlations above provide some evidence that reward benefits on WM are not simply due to more time spent making responses, it is still not definitive. We therefore applied the misbinding modelling to the mouse trajectories during responses. To compare the effects of reward across tasks that have different additional factors, we regressed each of these other factors out for each task separately (in Experiment 4 we only looked at the equal trials) leaving only residual effects of time and reward. We then ran regressions on each time point to get a p-value for the effect of reward at each time point, and ran time* reward regressions to see if the reward effect differed by time - these were done for each model parameter separately.

Time and reward significantly affected each parameter (Figure 7; $<<.05$ ), but only guessing rate had a reward * time interaction $(p=.0055)$, meaning that the effect of reward on imprecision, target responding and misbinding was the same across all time points.
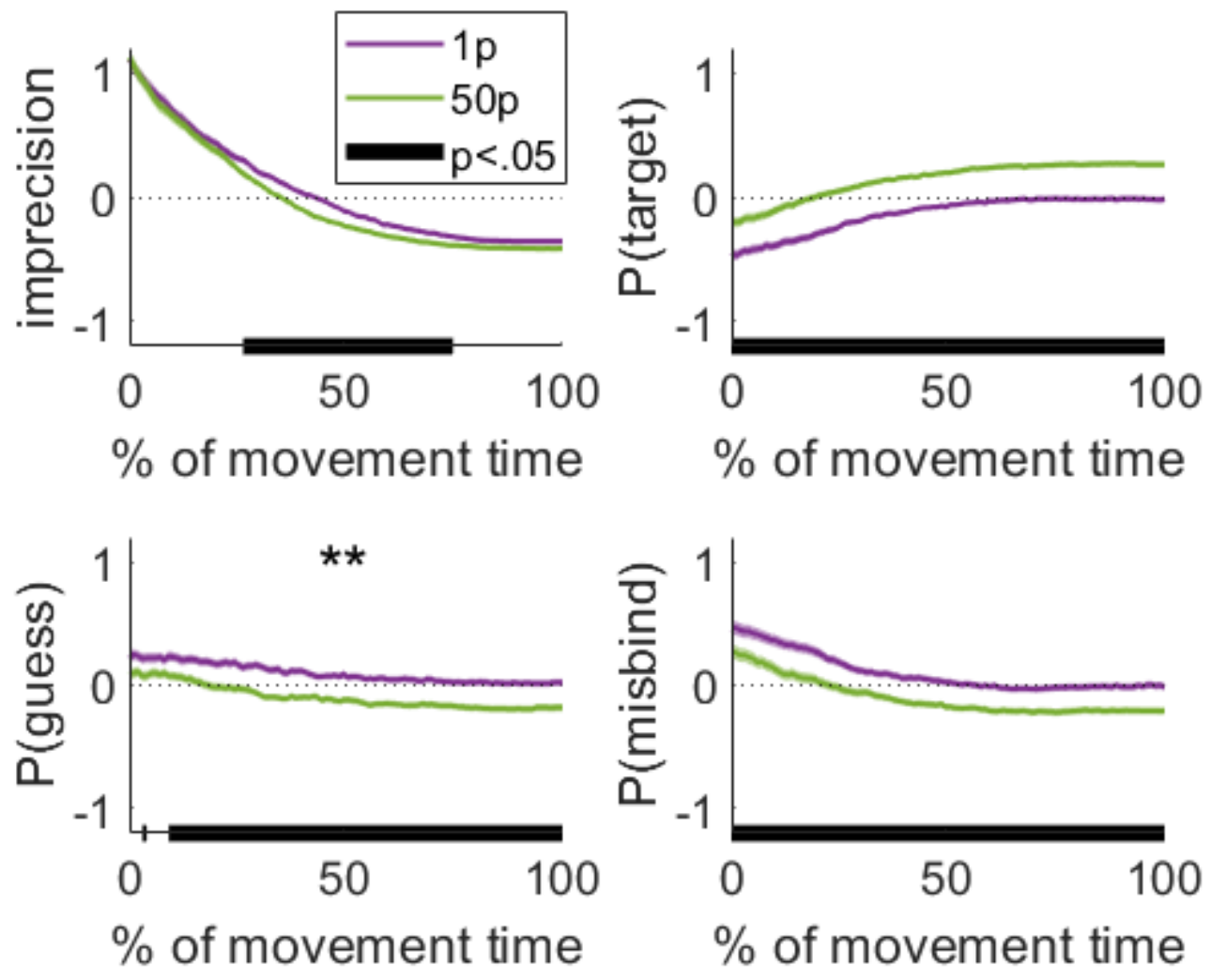

Figure 7. Mean mixture model parameter values at each percentage of the response movement. We interpolated 100 time points across the response trajectories, fit the misbinding model at each time point, and compared the residual reward effects (after regressing out any other factors e.g., cue-time, delay, congruency) for all the equal reward conditions across experiments 1-4, for each model parameter. The black bar at the bottom of each figure shows the time points with significant reward effects $(p<.05)$, while the stars at the top show which parameter had a significant reward*time interaction, indicating that the reward effect changed over time.

When we did a similar analysis for the unequal reward trials from experiment 4, it showed significant effects of reward, time and reward*time for all parameters, meaning that unequal reward effects differ across the response time. Imprecision benefits grew over time, while the other benefits shrank over time (this was likely due to initial trajectories in low unequal reward being biased towards the 
high-reward item). Importantly, there were reward*time*equal interactions for all parameters ( $p<$ .0001), indicating that these time* reward interactions differed when rewards were equal or unequal.

\section{General Discussion}

Using trial-wide rewards, rather than item-specific rewards, we showed that people can improve their working memory accuracy, and this process does not depend on when the reward-cue is presented. The same benefits were seen before or after encoding and maintenance, implicating retrieval mechanisms. The slower RT suggests a speed-accuracy trade-off, which was associated with greater target selection (via less guessing and/or misbinding) without changes in imprecision of responses. This suggests that when strategic prioritisation of WM capacity is not possible because all items are equally rewarded, people instead slow the item selection stage of retrieval down, in order to improve accuracy and maximise reward.

One explanation for this is that time during retrieval is costly, and that rewards motivate people to pay that cost to maximise rewards. There are many forms this cost could take, for example in a recent WM model retrieval is proposed to occur via sampling spikes from neurons storing the items, and greater accuracy can be achieved at the cost of longer sampling times and more energy spent on generating spikes (Schneegans, Taylor, \& Bays, 2020). Alternatively, time spent on one process is time not spent on another process, leading to opportunity costs for every cognitive function, which are balanced depending on the values associated with their outcomes (Kurzban, Duckworth, Kable, \& Myers, 2013; Shenhav et al., 2017). Our task had a fixed number of trials rather than a time limit, so slowing responses to increase accuracy may have been an optimal strategy to maximise rewards received, which may not always be the case in experimental tasks. Our work also challenges the resource-rational theory of WM (van den Berg \& Ma, 2018), which proposes a cost associated with using neural resources to encode items in WM, and predicts better encoding when rewards offset this cost.

The reward benefits were seen from the start of the response movement, suggesting that they were not solely due to more time spent fine-tuning the response to get it closer to the remembered orientation, and this is backed up by the lack of precision improvements and the independence of accuracy on the WM and motor-control tasks. This could reflect people initiating their responses when they have already selected the item, or could reflect some pre-threshold decision activity (Freeman, Dale, \& Farmer, 2011; Gallivan, Chapman, Wolpert, \& Flanagan, 2018; Jason, Chapman, \& Masson, 2014) which is more accurate when motivated.

The reward benefits did not affect the imprecision of the feature recall (orientation), but rather improved selection of the target, and this benefit was the same when items were already active in WM (via an incidental retro-cue). This suggests that reward did not facilitate the "loading" of an item into active WM to allow feature recall, and may suggest that the memory storage aspect of this task was unaffected by motivation. Previous studies have also found no benefit of reward on WM precision (van den Berg et al., 2020; Wallis et al., 2015), and others have found rewards and punishments affect item selection during WM (Fallon, Dolfen, Parolo, Zokaei, \& Husain, 2019; Wallis et al., 2015). Some of these tasks used trial-wide rewards, while others used item-specific rewards, and the motivational processes will depend heavily on this as well as when the rewards were known. When stimuli have different rewards, people can strategically choose to focus all their effort on only the high-reward ones, as increasing the accuracy of that item is worth enough to offset the loss of the low-reward. Such motivated prioritisation can improve feature precision, and has greatest effect when the rewards are shown before encoding (Allen \& Ueno, 2018; Atkinson et al., 2018; Klink et 
al., 2017). We also found that unequal rewards improved WM precision, and the mixture modelling and RT showed that this benefit depended on cue-time. When prioritisation occurred before the stimuli, people were quick to respond and more likely to guess when probed on the low-item, whereas if the prioritisation occurred after the stimuli, they were slower and more likely to misbind. Proactive prioritisation could include attentional resourcing - in this task, people knew where the items would appear so could even avoid looking at the low-reward item, preventing encoding entirely and forcing them to guess when probed on it. However, retroactive prioritisation means people must shift their resources to one item, which may have increased the misbinding of features between them. The fact that guessing was much lower for the low-reward item when post-cued suggests that it was not fully removed from memory, only de-prioritised.

Whether motivation decreases guessing or misbinding was unclear across these experiments, as some found effects on one or the other parameter. One explanation is that the misbinding parameter does not measure only true 'neural' misbinding (i.e. feature-swaps), but also picks up on educated guesses where participants can remember the orientations of non-target items but not the target and so respond close to those (Pratte, 2018). Thus, a process that leads to more guessing can also affect the 'misbinding' parameter in these models. Measuring confidence in participants' responses may be needed to distinguish these effects.

In conclusion, we found that people did not increase their WM capacity when motivated by equal rewards. Instead, they tuned their retrieval strategy to improve WM accuracy despite this limit. This is in keeping with reward paying a cost of time during the retrieval decision. This differs from the prioritisation strategy possible when rewards are unequal between memoranda, which preserves precision for one item's feature at the cost of much worse performance for other items, and may reflect different strategies depending on when the cue is given. These strategies are not mutually exclusive, as people can still slow down during retrieval for unequal rewards, and this demonstrates that motivation can still improve performance despite hard limits embedded in systems, and illustrates the flexibility in the WM system for goal-directed enhancement.

\section{Author Contributions}

JPG: conceptualisation, methodology, resources, investigation, data curation, analysis, visualisation, writing - original draft. GR: investigation. MK: investigation. SGM: conceptualisation, supervision, writing - review and editing.

\section{Acknowledgements}

We would like to thank the funders for their support (MRC Clinician Scientist Fellowship to SGM, $\mathrm{MR} / \mathrm{P00878X).}$

\section{Statement of Interests}

The authors declare no conflicts of interest regarding publication of this article.

\section{References}

Allen, R. J., \& Ueno, T. (2018). Multiple high-reward items can be prioritized in working memory but with greater vulnerability to interference. Attention, Perception, and Psychophysics, 80(7), 1731-1743. https://doi.org/10.3758/s13414-018-1543-6

Atkinson, A. L., Berry, E. D. J., Waterman, A. H., Baddeley, A. D., Hitch, G. J., \& Allen, R. J. (2018). Are there multiple ways to direct attention in working memory? Annals of the New York Academy of Sciences, 1424(1), 115-126. https://doi.org/10.1111/nyas.13634 
Bays, P. M., Catalao, R. F. G., \& Husain, M. (2009). The precision of visual working memory is set by allocation of a shared resource. Journal of Vision, 9(10), 7-7. https://doi.org/10.1167/9.10.7

Bays, P. M., \& Husain, M. (2008). Supplementary: Dynamic shifts of limited working memory resources in human vision. Science, 321(5890), 851-854.

https://doi.org/10.1126/science.1158023

Berridge, K. C. (2007). The debate over dopamine's role in reward: the case for incentive salience. Psychopharmacology, 191(3), 391-431. https://doi.org/10.1007/s00213-006-0578-x

Bogacz, R., Brown, E., Moehlis, J., Holmes, P., \& Cohen, J. D. (2006). The physics of optimal decision making: a formal analysis of models of performance in two-alternative forced-choice tasks. Psychological Review, 113(4), 700-765. https://doi.org/10.1037/0033-295X.113.4.700

Brown, S. D., \& Heathcote, A. (2008). The simplest complete model of choice response time: Linear ballistic accumulation. Cognitive Psychology, 57(3), 153-178.

https://doi.org/10.1016/j.cogpsych.2007.12.002

Burak, Y., \& Fiete, I. R. (2012). Fundamental limits on persistent activity in networks of noisy neurons. Proceedings of the National Academy of Sciences, 114(20), E4117-E4117. https://doi.org/10.1073/pnas.1706051114

Codol, O., Holland, P. J., Manohar, S. G., \& Galea, J. M. (2019). Reward-based improvements in motor control are driven by multiple error-reducing mechanisms. BioRxiv. https://doi.org/10.1101/792598

Fallon, S. J., Dolfen, N., Parolo, F., Zokaei, N., \& Husain, M. (2019). Task-irrelevant financial losses inhibit the removal of information from working memory. Scientific Reports, 9(1), 1-9. https://doi.org/10.1038/s41598-018-36826-x

Freeman, J. B., Dale, R., \& Farmer, T. A. (2011). Hand in motion reveals mind in motion. Frontiers in Psychology, 2(April), 1-6. https://doi.org/10.3389/fpsyg.2011.00059

Gallivan, J. P., Chapman, C. S., Wolpert, D. M., \& Flanagan, J. R. (2018). Decision-making in sensorimotor control. Nature Reviews Neuroscience 2018, 1. https://doi.org/10.1038/s41583018-0045-9

Hitch, G. J., Hu, Y., Allen, R. J., \& Baddeley, A. D. (2018a). Competition for the focus of attention in visual working memory: perceptual recency versus executive control. Annals of the New York Academy of Sciences, 1424(1), 64-75. https://doi.org/10.1111/nyas.13631

Hitch, G. J., Hu, Y., Allen, R. J., \& Baddeley, A. D. (2018b). Competition for the focus of attention in visual working memory: Perceptual recency versus executive control. Annals of the New York Academy of Sciences, 1424(1), 64-75. https://doi.org/10.1111/nyas.13631

Hu, Y., Allen, R. J., Baddeley, A. D., \& Hitch, G. J. (2016). Executive control of stimulus-driven and goal-directed attention in visual working memory. Attention, Perception, and Psychophysics, 78(7), 2164-2175. https://doi.org/10.3758/s13414-016-1106-7

Hu, Y., Hitch, G. J., Baddeley, A. D., Zhang, M., \& Allen, R. J. (2014). Executive and perceptual attention play different roles in visual working memory: Evidence from suffix and strategy effects. Journal of Experimental Psychology: Human Perception and Performance, 40(4), 16651678. https://doi.org/10.1037/a0037163

Jason, P., Chapman, C. S., \& Masson, M. E. J. (2014). Three-dimensional reach trajectories as a probe of real-time decision-making between multiple competing targets. Frontiers in Neuroscience, 8(251), 1-19. https://doi.org/10.3389/fnins.2014.00215 
Klink, P. C., Jeurissen, D., Theeuwes, J., Denys, D., \& Roelfsema, P. R. (2017). Working memory accuracy for multiple targets is driven by reward expectation and stimulus contrast with different time-courses. Scientific Reports, 7(1), 1-13. https://doi.org/10.1038/s41598-01708608-4

Kurzban, R., Duckworth, A., Kable, J. W., \& Myers, J. (2013). An opportunity cost model of subjective effort and task performance. Behavioral and Brain Sciences, 36(6), 661-679. https://doi.org/10.1017/S0140525X12003196

Manohar, S. G., Chong, T. T. J., Apps, M. A. J., Batla, A., Stamelou, M., Jarman, P. R., ... Husain, M. (2015). Reward Pays the Cost of Noise Reduction in Motor and Cognitive Control. Current Biology, 25(13), 1707-1716. https://doi.org/10.1016/j.cub.2015.05.038

Manohar, S. G., Muhammed, K., Fallon, S. J., \& Husain, M. (2019). Motivation dynamically increases noise resistance by internal feedback during movement. Neuropsychologia, 123, 19-29. https://doi.org/10.1016/j.neuropsychologia.2018.07.011

Manohar, S. G., Zokaei, N., Fallon, S. J., Vogels, T. P., \& Husain, M. (2019). Neural mechanisms of attending to items in working memory. Neuroscience and Biobehavioral Reviews, 101(January), 1-12. https://doi.org/10.1016/j.neubiorev.2019.03.017

Miller, G. A. (1956). The magical number seven, plus or minus two: some limits on our capacity for processing information. 1956. Psychological Review, 101(2), 343-352. https://doi.org/10.1037/h0043158

Morey, C. C., Cowan, N., Morey, R. D., \& Rouder, J. N. (2011). Flexible attention allocation to visual and auditory working memory tasks: Manipulating reward induces a trade-off. Attention, Perception, and Psychophysics, 73(2), 458-472. https://doi.org/10.3758/s13414-010-0031-4

Pearson, B., Raskevicius, J., Bays, P. M., Pertzov, Y., \& Husain, M. (2014). Working memory retrieval as a decision process. Journal of Vision, 14(2), 2-2. https://doi.org/10.1167/14.2.2

Pratte, M. S. (2018). Swap errors in spatial working memory are guesses. Psychonomic Bulletin and Review. https://doi.org/10.3758/s13423-018-1524-8

Schneegans, S., \& Bays, P. M. (2016). No fixed item limit in visuospatial working memory. Cortex, 83, 181-193. https://doi.org/10.1016/j.cortex.2016.07.021

Schneegans, S., \& Bays, P. M. (2018). Drift in Neural Population Activity Causes Working Memory to Deteriorate Over Time. The Journal of Neuroscience, 38(21), 4859-4869.

https://doi.org/10.1523/JNEUROSCI.3440-17.2018

Schneegans, S., Taylor, R., \& Bays, P. M. (2020). Stochastic sampling provides a unifying account of visual working memory limits. Proceedings of the National Academy of Sciences. Retrieved from https://www.pnas.org/content/early/2020/08/10/2004306117?s=09

Shen, W., Flajolet, M., Greengard, P., \& Surmeier, D. J. (2008). Dichotomous dopaminergic control of striatal synaptic plasticity. Science, 321(5890), 848-851. https://doi.org/10.1126/science.1160575

Shenhav, A., Musslick, S., Lieder, F., Kool, W., Griffiths, T. L., Cohen, J. D., \& Botvinick, M. M. (2017). Toward a Rational and Mechanistic Account of Mental Effort. Annual Review of Neuroscience, 40(1), 99-124. https://doi.org/10.1146/annurev-neuro-072116-031526

Souza, A. S., \& Oberauer, K. (2016). In search of the focus of attention in working memory: 13 years of the retro-cue effect. Attention, Perception, and Psychophysics, 78(7), 1839-1860. https://doi.org/10.3758/s13414-016-1108-5 
Stokes, M. G. (2015). 'Activity-silent' working memory in prefrontal cortex: a dynamic coding framework. Trends in Cognitive Sciences, 19(7), 394-405.

https://doi.org/10.1016/j.tics.2015.05.004

van den Berg, R., \& Ma, W. J. (2018). A resource-rational theory of set size effects in human visual working memory. ELife, 7, 1-31. https://doi.org/10.7554/eLife.34963

van den Berg, R., Shin, H., Chou, W.-C., George, R., \& Ma, W. J. (2012). Variability in encoding precision accounts for visual short-term memory limitations. Proceedings of the National Academy of Sciences of the United States of America, 109(22), 8780-8785. https://doi.org/10.1073/pnas.1117465109

van den Berg, R., Zou, Q., \& Ma, W. J. (2020). No effect of monetary reward in a visual working memory task. BioRxiv, 767343. https://doi.org/10.1101/767343

Vijayraghavan, S., Wang, M., Birnbaum, S. G., Williams, G. V, \& Arnsten, A. F. T. (2007). Inverted-U dopamine $D 1$ receptor actions on prefrontal neurons engaged in working memory. Nature Neuroscience, 10(3), 376-384. https://doi.org/10.1038/nn1846

Wallis, G., Stokes, M. G., Arnold, C., \& Nobre, A. C. (2015). Reward boosts working memory encoding over a brief temporal window. Visual Cognition, 23(1-2), 291-312. https://doi.org/10.1080/13506285.2015.1013168

Yee, D. M., \& Braver, T. S. (2018). Interactions of motivation and cognitive control. Current Opinion in Behavioral Sciences, 19, 83-90. https://doi.org/10.1016/j.cobeha.2017.11.009 